\title{
Knockdown of S100P by lentiviral-mediated RNAi promotes apoptosis and suppresses the colony-formation ability of gastric cancer cells
}

\author{
QI ZHANG ${ }^{1,2}$, HAOLIN HU $^{1}$, XIN SHI $^{1}$ and WENHAO TANG ${ }^{1}$ \\ ${ }^{1}$ Department of General Surgery, Zhongda Hospital, Southeast University; \\ ${ }^{2}$ Medical College, Southeast University, Gulou, Nanjing, Jiangsu 210009, P.R. China
}

Received January 23, 2014; Accepted March 6, 2014

DOI: $10.3892 /$ or.2014.3104

\begin{abstract}
S100P is a putative candidate oncogene in several types of human tumors. However, expression of S100P, its potential role and its clinical significance in gastric cancer remain unclear. In the present study, S100P expression was examined by immunohistochemistry using a tissue microarray. Positive staining for S100P was noted in $77.1 \%$ of the cases while $22.9 \%$ were negative. In two gastric cancer cell lines, MGC-803 and SGC-7901, S100P expression was knocked down by a lentiviral short hairpin delivery system. The RNA interference-mediated downregulation of S100P expression markedly promoted cell apoptosis and inhibited cell colony-formation ability of the gastric cancer cells. In addition, knockdown of S100P significantly regulated the expression of 12 apoptosis-associated genes with a $>1.5$-fold change compared with the negative control. Among them, FOS, DDIT3 and FN1 were significantly upregulated, while FASLG, DAPK1, CTNNB1 and CASP2 were notably downregulated following S100P silencing. These results suggest that $\mathrm{S} 100 \mathrm{P}$ acts as an oncogenic factor in gastric cancer and is a potential molecular target for gastric cancer gene therapy.
\end{abstract}

\section{Introduction}

Gastric cancer (GC) is the fourth most common malignancy and the second leading cause of cancer-related mortality in men worldwide (1). A total of 989,600 new cases of GC were diagnosed and 738,000 GC-related deaths occurred in 2008, accounting for $8 \%$ of the total cases and $10 \%$ of total deaths.

Correspondence to: Dr Xin Shi, Department of General Surgery, Zhongda Hospital, Southeast University, No. 87 Dingjiaqiao, Gulou, Nanjing, Jiangsu 210009, P.R. China

E-mail: dnshixin@126.com

Abbreviations: GC, gastric cancer, RNAi, RNA interference; TMA, tissue microarray; shRNA, short hairpin RNA; FACS, fluorescence-activated cell sorter

Key words: gastric cancer, S100P, lentivirus, shRNA, apoptosis
More than 2/3 of new cases and deaths occur in developing countries, with the highest frequency in Eastern Asia (2). In spite of multi-treatments including surgery, chemotherapy, radiotherapy, and molecular-targeted therapy, the overall 5-year survival rate of GC patients remains extremely low. In the United States where fewer GCs are diagnosed at an early stage, the 5-year survival rate of stomach cancer is only $\sim 24 \%$ (1). The crude mortality rate of GC in China was 25.2 per 100,000 individuals, which accounted for $23.2 \%$ of the total cancer-related deaths in 1990-1992 (3). Therefore, there is an urgent need to elucidate the molecular mechanisms of GC and to develop novel therapeutic strategies to treat GC.

Dysregulation of gene expression is associated with the neoplastic process, and there is compelling evidence implicating differential expression of multiple genes in the development and progression of GC. S100P belongs to the S100 family of proteins. S100 is a family of calcium-binding proteins characterized by EF-hand motifs. The name S100 is due to the $100 \%$ solubility of these proteins in a $100 \%$ saturated ammonium sulphate solution. The S100 proteins form homodimers or heterodimers and are involved in multifunctional cellular activities including protein phosphorylation, gene transcription, cell proliferation, survival and apoptosis (4). S100P is one of the least studied members of this family. S100P is a 95-amino acid protein, which was first purified and characterized from placenta by Becker et al (5) in 1992. Recently, studies indicate that $\mathrm{S} 100 \mathrm{P}$ is overexpressed in a variety of cancers, including pancreatic (6), colon (7), breast (8) and lung carcinomas (9), and its expression has been shown to be associated with poor clinical outcomes. In pancreatic and colon cancer, S100P was found to promote proliferation, invasion and migration of cancer via the receptor for activated glycation end products (RAGE) (6,7). S100P levels have been correlated with poor patient survival in breast (8) and lung cancer (9). In addition, intracellular S100P binds to the N-terminal domain of dormant ezrin, resulting in the activation of ezrin and stimulation of the transendothelial migration of tumor cells (10). Furthermore, the interaction between S100P and calcyclin-binding protein and Siah-1-interacting protein (CacyBP/SIP) was found to lead to the degradation of $\beta$-catenin, which is involved in the tumorigenesis for many different types of cancers (11). GC cells exhibit an increase in S100P expression following the treatment of all-trans retinoic acid (12). However, the 
functional roles of S100P in cancer progression, including GC, remain to be elucidated.

In the present study, we applied RNA interference (RNAi) technology to knock down the expression of S100P in two GC cell lines MGC-803 and SGC-7901, and we investigated the apoptosis rate and colony-formation capacity in both cell lines. Subsequently, changes in the expression of apoptosisrelated genes following S100P downregulation were detected. Our data revealed that the inhibition of S100P significantly promoted apoptosis and suppressed colony-formation ability in both cell lines. S100P was significantly involved in the regulation of apoptosis-related gene expression. Thus, S100P appears to be a potential target for gene therapy in GC.

\section{Materials and methods}

Patients and tissue microarray. In the present study, the GC tissue microarray (TMA) was purchased from Fanpu Biotech, Inc. (Guilin China), which contains 198 GCs (adenocarcinoma, mucinous adenocarcinoma, signet ring cell carcinoma and undifferentiated carcinoma). All of the tissues were obtained from archives of paraffin-embedded tissues between 2005 and 2008. The patient with GCs included 146 males and 52 females. Ages of the 198 patients with gastric carcinoma ranged from 24 to 83 years (mean age, 58.1 years). Patient consent was obtained, along with approval from the local medical ethics committee, for the use of human tissue samples for research purposes.

Immunohistochemical staining. Immunohistochemistry (IHC) was performed using a standard streptavidin biotinperoxidase complex method. Consecutive sections were deparaffinized with xylene and dehydrated with alcohol. Endogenous peroxidase was quenched with $3 \% \mathrm{H}_{2} \mathrm{O}_{2}$ in $1 \mathrm{X}$ PBS. For antigen retrieval, TMA slides were treated in $10 \mathrm{mM}$ citrate buffer ( $\mathrm{pH}$ 6.0) and boiled by electronic wave or microwave for $10 \mathrm{~min}$. Bovine serum albumin $5 \%$ was then applied for $20 \mathrm{~min}$ at $37^{\circ} \mathrm{C}$ to prevent non-specific binding. The TMA slides were incubated with anti-S100P (1:250 dilution; Abcam, Cambridge, UK) in a moist chamber overnight at $4{ }^{\circ} \mathrm{C}$, followed by HRP-conjugated anti-rabbit secondary antibodies (Shanghai Weiao Ltd., Shanghai, China) for $20 \mathrm{~min}$ at $37^{\circ} \mathrm{C}$. After each treatment, the slides were washed with PBS ( $\mathrm{pH} 7.2-7.6)$ three times for $2 \mathrm{~min}$. All slides were colored with the DAB substrate chromogen system (Dako Co.) and counterstained with Mayer's hematoxylin. Omission of the primary antibody was used as a negative control, and appropriate positive controls were used as recommended by the manufacturers. Slides were analyzed independently by two pathologists using fluorescence microscopy. Photographs were captured using a Nikon digital still camera. The sections were evaluated by Aperio ImageScope, and the number of positive cells was calculated at x200 magnification.

Evaluation of immunohistochemistry. One hundred cells were randomly selected and counted from five representative fields of each section by two independent pathologists blinded to the clinicopathologic information. The proportion of positively staining cells was evaluated according to five levels: $<1 \%$, negative; $1-5 \%$, borderline; $6-25 \%$, intermediate; $26-50 \%$, moderate; $>50 \%$ of the carcinoma cells stained, strong (8). For each core, the intensity of the tumor immunoreactivity was evaluated on a scale from 0 to 3 based on the method by Allred et al (13): 0, negative; 1, detectable but weak; 2, moderate but submaximal; or 3, maximal. Staining scores for S100P were calculated as the sum of each intensity multiplied by the percentage of positive cells, giving a range from 0 $(0 \times 100 \%)$ to $300(3 \times 100 \%)$. For statistical purposes, the IHC scores were arbitrarily divided into two groups: a staining score $\leq 18$ was considered as negative and a staining score $>18$ was considered as positive.

Cell culture and infection. The following human gastric adenocarcinoma cell lines were employed: adenocarcinoma cell line MGC-803 purchased from the Shanghai Cell Bank (Shanghai, China) and SGC-7901 preserved at the Medical College of Southeast University (Nanjing, China). The cell lines were maintained in RPMI-1640 medium containing 10\% fetal bovine serum (FBS; Gibco), $2 \mathrm{mM}$ L-glutamine and $1 \%$ penicillin/streptomycin mixture at $37^{\circ} \mathrm{C}$ in a humidified atmosphere of $5 \% \mathrm{CO}_{2}$ and $95 \%$ air.

For the lentiviral infection, MGC-803 and SGC-7901 cells were cultured in 6-well plates. The S100P short hairpin RNA (shRNA) expressing the lentivirus (sh-S100P) or non-targeting shRNA expressing GFP (negative control) were added, with a multiplicity of infection of 100 in the MGC-803 and 20 in the SGC-7901 cells, respectively. After $72 \mathrm{~h}$ of infection, cells were observed using fluorescence microscopy (Olympus, Tokyo, Japan).

Lentiviral vector production. Five small interfering RNAs (siRNAs) targeting the sequence of S100P were transformed into shRNAs with stem-loop-stem structure and were cloned into GV115-hU6-EGFP-lentiviral vectors with AgeI and EcoRI sites. The positive recombinant clone was identified by PCR and DNA sequencing. The effective targeting sequence of S100P (TCA GTG AGT TCA TCG TGT T) was selected by western blot analysis. A non-silencing shRNA sequence (TTC TCC GAA CGT GTC ACG T) was used as a negative control. The recombined GV115-S100P-lentiviral vector or the negative control lentiviral vector plasmid and pHelper 1.0 plasmid, the pHelper 2.0 plasmid (Shanghai GeneChem Co., Ltd., Shanghai, China) were cotransfected into HEK293T cells via Lipofectamine 2000 (Invitrogen, Carlsbad, CA, USA) to generate the lentivirus. After 3 days of incubation, the lentivirus from the culture medium was collected and concentrated with Centricon Plus-20 (Millipore, Billerica, MA, USA). The concentrated viral supernatant was aliquoted and kept at $-80^{\circ} \mathrm{C}$ before use.

Real-time PCR. MGC-803 and SGC-7901 cells were cultured in 6-well plates and were then infected with the lentivirus for 96 and $120 \mathrm{~h}$, respectively. Total RNA was isolated from the cultured cells using TRIzol reagent (Invitrogen). cDNA was generated by reverse transcription from total mRNA. Two sets of primers were used for real-time PCR. Primers for S100P and GAPDH were designed by Primer Premier software as follows: S100P-F, 5'-AGG AAG GTG GGT CTG AAT CTA-3' and S100P-R, 5'-TCC ACG GCA TCC TTG TCT-3'; GAPDH-F, 5'-TGA CTT CAA CAG CGA CAC CCA-3' and 
GAPDH-R, 5'-CAC CCT GTT GCT GTA GCC AAA-3'. The SYBR-Green Real-Time PCR assay kit (Takara, Bio Inc., Otsu, Japan) was used, and quantitative real-time PCR (qRTPCR) was performed according to the ABI manufacturer's protocols (Applied Biosystems, Foster City, CA, USA). All samples were examined in triplicate.

The 20- $\mu \mathrm{l}$ reaction mixture contained $10 \mu \mathrm{l}$ SYBR-Green PCR Master Mix (Takara), $1 \mu \mathrm{l}$ cDNA template, $0.5 \mu \mathrm{l} \mathrm{PCR}$ forward primer, $0.5 \mu \mathrm{l}$ PCR reverse primer and $8.0 \mu \mathrm{l}$ RNasefree $\mathrm{H}_{2} \mathrm{O}$. PCR running conditions consisted of $30 \mathrm{sec}$ at $95^{\circ} \mathrm{C}$ for the initial denaturation, 45 cycles of $5 \mathrm{sec}$ at $95^{\circ} \mathrm{C}$ and $30 \mathrm{sec}$ at $60^{\circ} \mathrm{C}$. The melting curve was monitored with the following conditions: $15 \mathrm{sec}$ at $95^{\circ} \mathrm{C}, 30 \mathrm{sec}$ at $55^{\circ} \mathrm{C}$, followed by a temperature range from $55^{\circ} \mathrm{C}$ to $95^{\circ} \mathrm{C}$ increased by $0.5^{\circ} \mathrm{C}$ every $4 \mathrm{sec}$. GAPDH was employed as an internal reference under the same experimental conditions. The threshold cycle (Ct values), which was the cycle number at which the amount of the amplified gene of interest reached a fixed threshold, was subsequently determined. Relative quantification of the S100P mRNA levels was normalized to human GAPDH levels and calculated with the $2^{-\Delta \Delta \mathrm{Ct}}$ method (14).

Western blot analysis. MGC-803 and SGC-7901 cells were cultured in 6-well plates and were then infected with the lentivirus for 168 and $96 \mathrm{~h}$, respectively. Total protein was isolated from whole cells via ice-cold protein lysis buffer (100 mM Tris- $\mathrm{HCl}, \mathrm{pH} 6.8,2 \%$ mercaptoethanol, 4\% SDS, $20 \%$ glycerine $1,0.01 \%$ bromcresol blue), followed by $15 \mathrm{~min}$ of incubation on ice and centrifugation at $12,000 \mathrm{x} \mathrm{g}$ for $5 \mathrm{~min}$ at $4^{\circ} \mathrm{C}$. The protein concentration was determined by BCA protein assay (Pierce, Rockford, IL, USA). Protein extracts were separated on a SDS-polyacrylamide gel (12\%), blotted onto a PVDF membrane and incubated with the antiS100P antibody (1:1,000; Abcam, Cambridge, UK) or the anti-GAPDH antibody (1:5,000; Santa Cruz Biotechnology, Santa Cruz, CA, USA). Western blotting was developed using horseradish peroxidase-conjugated goat anti-mouse or goat anti-rabbit IgG (1:5,000; Santa Cruz Biotechnology). The membranes were detected with enhanced chemiluminescence reagent (Amersham, Braunschweig, Germany) and recorded on film in the linear detectable range.

Colony-formation assay. Cells were seeded in 12-well plates at a density of 300 cells/well and incubated at $37^{\circ} \mathrm{C}$ for 14 days to form colonies. The medium was replaced every 2 days. At the indicated time-point, cells were washed twice with PBS, fixed with paraform for $1 \mathrm{~h}$, stained with Giemsa for $20 \mathrm{~min}$, washed with $\mathrm{ddH}_{2} \mathrm{O}$ three times and then photographed with a digital camera. The number of colonies ( $>50$ cells/colony) was counted using fluorescence microscopy (MicroPublisher 3.3 RTV; Olympus).

Analysis of apoptosis as determined by fluorescence-activated cell sorter (FACS). Analysis of apoptosis was performed with the Annexin V-APC apoptosis detection kit according to the manufacturer's instructions (88-8007; eBioscience). Cells were seeded and infected with the lentivirus. The cells were washed once with PBS and centrifugated at $1500 \mathrm{x} g$ for $5 \mathrm{~min}$, washed once in $1 \mathrm{X}$ binding buffer and resuspended in $1 \mathrm{X}$ staining buffer to $10^{6}-10^{7}$ cells $/ \mathrm{ml}$. Furthermore,
Table I. S100P expression in the TMA of GC tissues.

\begin{tabular}{lccc}
\hline & \multicolumn{3}{c}{ S100P } \\
\cline { 2 - 4 } & Negative & Moderate & Strong \\
\hline Score interval & $(0,18)$ & $(18,90)$ & $(90,300)$ \\
Mean \pm SD & $2.204 \pm 4.063$ & $57.763 \pm 15.670$ & $129.639 \pm 40.565$ \\
No. of cases & 38 & 38 & 90 \\
Percentage & $22.90 \%$ & $22.90 \%$ & $54.2 \%$ \\
\hline
\end{tabular}

$100 \mu \mathrm{l}$ of the cells $\left(\sim 10^{5}-10^{6}\right.$ cells) was incubated with $5 \mu \mathrm{l}$ Annexin V-APC at room temperature for 10-15 $\mathrm{min}$ in the dark. Annexin V-stained cells were assessed using FACS flow cytometer (FACSCalibur; Becton-Dickinson).

Real-time PCR gene microarray assay. Differential expression of apoptosis-related genes was analyzed using the human apoptosis PCR array (Shanghai GeneChem). RNA isolation, DNase treatment, and RNA clean-up were performed according to the manufacturer's protocol (Qiagen, Hilden, Germany). The isolated RNA was reverse transcribed into cDNA using the RT2 First Strand kit (Invitrogen). The template was added to an instrument-specific, ready-to-use RT2 SYBR-Green qPCR Master Mix (Invitrogen). PCR was performed on a Takara TP800 instrument (Takara). Each assay was conducted in triplicate. Data normalization was based on correcting all $\mathrm{Ct}$ values for the average $\mathrm{Ct}$ values of several constantly expressed housekeeping genes present on the array. The threshold cycle (Ct) values for all the genes on each PCR array were calculated using the instrument-specific software, and the fold-changes in gene expression for pair-wise comparison were calculated using the $2^{-\Delta \Delta \mathrm{Ct}}$ method. The analysis was completed by Shanghai GeneChem.

Statistical analysis. The data shown are presented as the mean \pm standard deviation (SD). Each experiment was repeated at least three times. All statistical analyses were performed using SPSS v17.0 software (SPSS, Inc., Chicago, IL, USA). Student's t-test was used for comparison of data obtained between the control and test group. The significance was conventionally set at $\mathrm{P}$-value $<0.05$.

\section{Results}

S100P is frequently expressed in GC tissues. S100P expression was able to be evaluated in the TMA for 166/198 gastric carcinoma samples. The exclusion criteria included unrepresentative samples, no tumor tissue or samples with too few tumor cells ( 300 cells/case) and lost samples. The intensity of immunoreactivity ranged from negative to strong (Fig. 1). Of the 166 GCs in the TMA evaluated, 38 were negative, and the remaining 128 were classified into the positive group including moderate and strong expression subgroups (Table I). In addition, staining for S100P was found both in the cytoplasm and in the nucleus in $77.1(128 / 166)$ and $7.7 \%(13 / 166)$ of cases, respectively. 

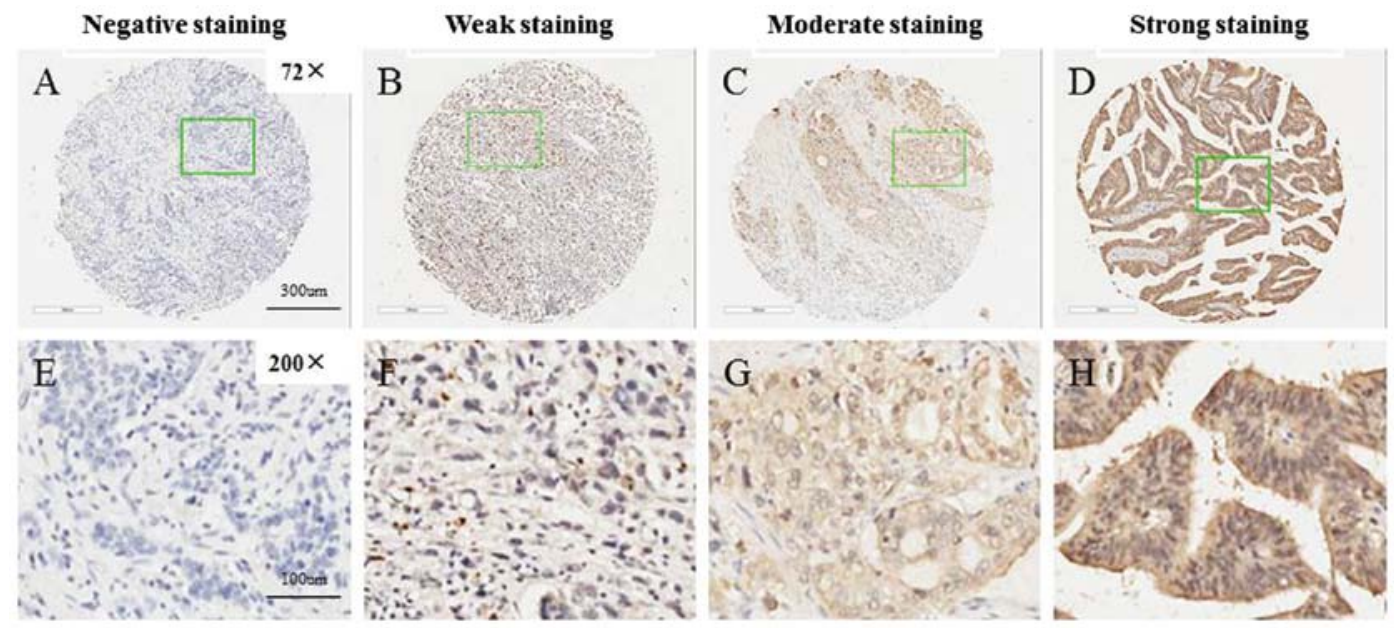

Figure 1. Representative images of S100P immunohistochemical staining in human GC tissue samples. (A and E) Negative staining, (B and F) weak staining, (C and G) moderately positive staining, (D and H), strongly positive staining. Original magnification x72 for A-D; x200 for E-H. The scale bar indicates 300 and $100 \mu \mathrm{m}$, respectively. The images were collected using Aperio ImageScope.

A
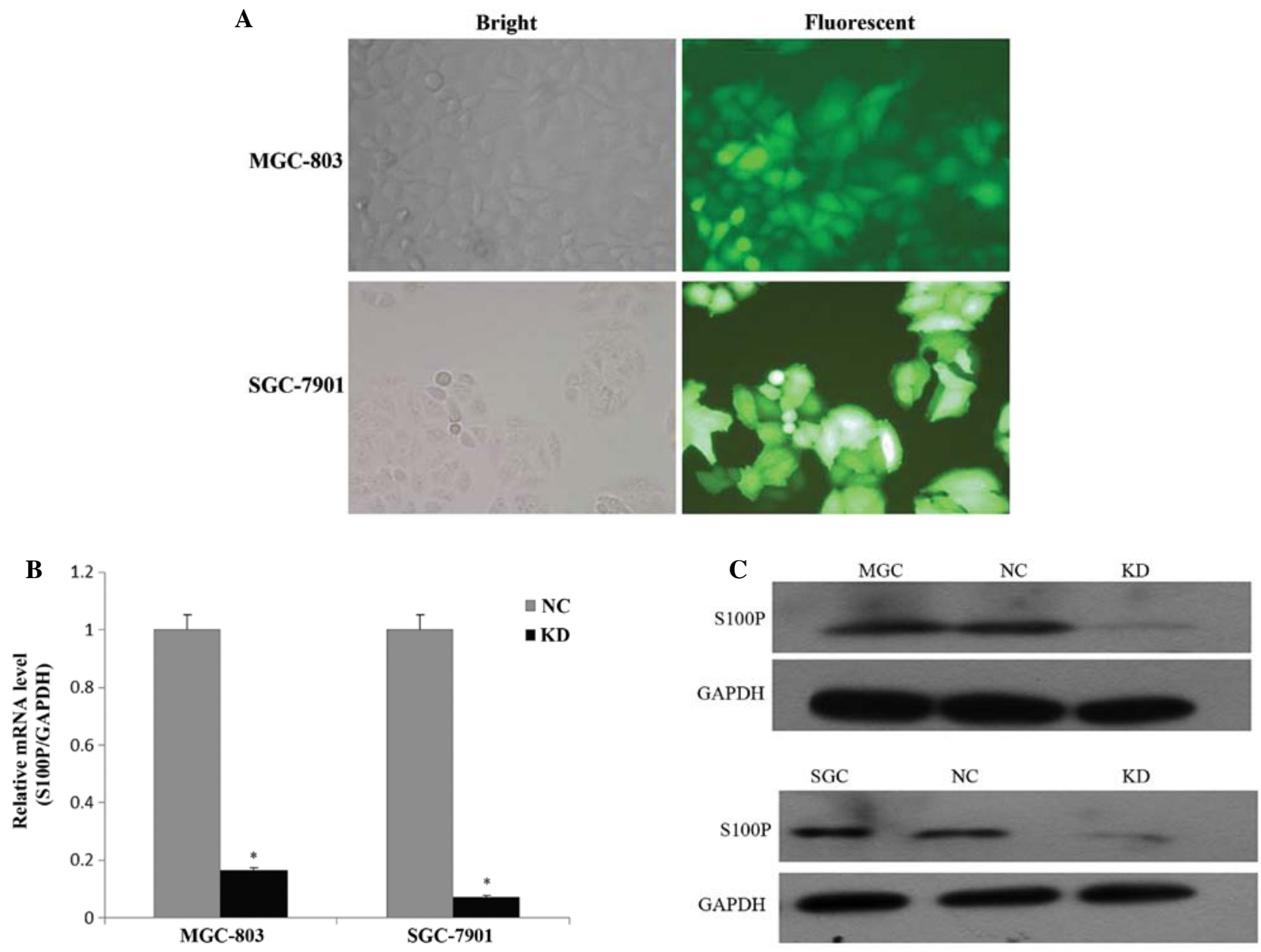

Figure 2. S100P silencing efficiency by shRNA lentivirus. (A) Lentivirus infection in GC cell lines. Fluorescence photomicrographs of gastric cancer cells infected by the lentivirus. Images were captured $72 \mathrm{~h}$ after infection at a magnification of x200. (B) Identification of S100P knockdown efficiency using shRNA lentivirus by real-time PCR in MGC-803 and SGC-7901 cells. (C) Identification of the S100P knockdown efficiency using shRNA lentivirus by western blot analysis. Reduced S100P protein levels in the MGC-803 and SGC-7901 cells are shown. GAPDH was used as a loading control (NC, negative control; KD, knockdown).

Knockdown of S100P by shRNA lentivirus system in GC cells. To investigate the role of S100P in GC, shRNA targeting $\mathrm{S} 100 \mathrm{P}$ or non-silencing sequences were cloned into the GV-115 lentiviral vector. Then, the S100P shRNA lentivirus or nonsilencing shRNA lentivirus expressing GFP were generated and infected into two GC cell lines with a high multiplicity of 

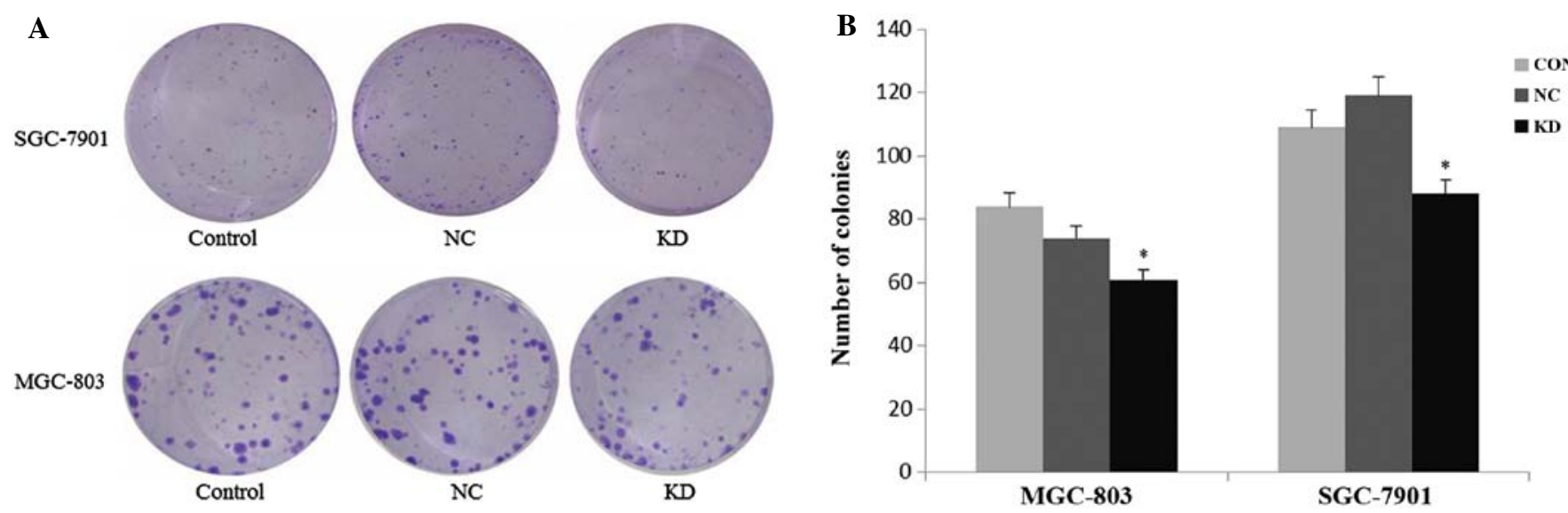

Figure 3. S100P silencing inhibits GC cell colony formation. (A) Photomicrographs of Giemsa-stained colonies of MGC-803 and SGC-7901 cells growing in 12-well plates for 14 days after infection with S100P shRNA lentivirus. (B) The number of cells in each colony in the GC cell lines was counted. The cell number in the knockdown group was significantly reduced, as compared with the control group (CON, uninfected cells; NC, negative control; KD, knockdown).

Table II. Candidate genes whose expression is altered in the S100P knockdown vs. the control group.

\begin{tabular}{llrrr}
\hline Gene & \multicolumn{1}{c}{ Description } & Gene ID & Fold-change & P-value \\
\hline S100P & S100 calcium-binding protein P & 6286 & -4.02102 & 0.0052 \\
NFAT5 & Nuclear factor of activated T-cells 5 & 10725 & -2.76273 & 0.0002 \\
FGFR3 & Fibroblast growth factor receptor 3 & 2261 & -2.13584 & 0.0001 \\
CASP2 & Caspase-2, apoptosis-related cysteine peptidase & 835 & -1.95844 & 0.0006 \\
STAT2 & Signal transducer and activator of transcription 2 & 6773 & -1.90022 & 0.0054 \\
FASLG & Fas ligand & 356 & -1.86964 & 0.0401 \\
HSPA1A & Heat shock protein 1A & 3303 & -1.72966 & 0.0021 \\
DAPK1 & Death-associated protein kinase 1 & 1612 & -1.67947 & 0.0009 \\
CTNNB1 & Catenin (cadherin-associated protein), $\beta 1$ & 1499 & -1.65682 & 0.0002 \\
TNFRSF25 & Tumor necrosis factor receptor superfamily, member 25 & 8718 & -1.61876 & 0.0122 \\
FOS & FBJ murine osteosarcoma viral oncogene homolog & 2353 & 1.54247 & 0.0024 \\
FN1 & Fibronectin 1 & 2335 & 1.75283 & 0.0000 \\
DDIT3 & DNA-damage-inducible transcript 3, also known as CHOP, GADD153 & 1649 & 2.70239 & 0.0001 \\
\hline
\end{tabular}

Of the 86 apoptosis-related genes analyzed, mRNA transcript levels of 12 genes were significantly altered as compared with the negative control. A positive number indicates upregulation while a negative number represents downregulation.

infection of 100 in the MGC-803 and 20 in the SGC-7901 cells, respectively. As shown in Fig. 2A, the infection efficiency of the lentivirus was $>80 \%$ after $72 \mathrm{~h}$ of infection. The qRT-PCR assay revealed that S100P mRNA expression in the GC cells treated with the S100P shRNA lentivirus was decreased by $83.4 \%$ in the MGC-803 and $92.7 \%$ in the SGC-7901 cells $(\mathrm{P}<0.05)$, when compared with the negative control group (Fig. 2B). We also determined the level of S100P protein in the MGC-803 and SGC-7901 cells after 168 and $96 \mathrm{~h}$ of lentiviral infection by western blot analysis (Fig. 2C). In the MGC-803 and SGC-7901 cell lines, the protein expression of S100P was significantly reduced by $\sim 80 \%$ following S100P shRNA lentivirus treatment $(\mathrm{P}<0.05)$.

Downregulation of S100P inhibits cell-colony formation. We then assessed the colony-formation capacity of MGC-803 and SGC-7901 cells treated with the S100P shRNA lentivirus. Three groups of MGC-803 and SGC-7901 cells (control, negative control and knockdown) were allowed to grow for 14 days to form colonies. As shown in Fig. 3, S100P knockdown resulted in a notable decrease in the number of colonies, as compared with the two control groups $(\mathrm{P}<0.05)$. The average colony number in the MGC-803 and SGC-7901 cells was $61 \pm 10$ and $88 \pm 8$, with nearly a 0.27 -fold and 0.19 -fold decrease, respectively, in the knockdown group compared with the negative control group.

Knockdown of S100P promotes the apoptosis of gastric cancer cells. To study the function of S100P on gastric cell apoptosis, MGC-803 and SGC-7901 cells were analyzed with FACS and Annexin V-APC staining. As shown in Fig. 4, a significant induction in apoptosis was noted in the S100P shRNA group compared to the control and negative control groups both in the MGC-803 and SGC-7901 cells $(\mathrm{P}<0.05)$. The percentage of apoptotic cells (M1) was increased to 9.12 and $4.96 \%$ in the MGC-803 and SGC-7901 cells, respectively. These data 
A
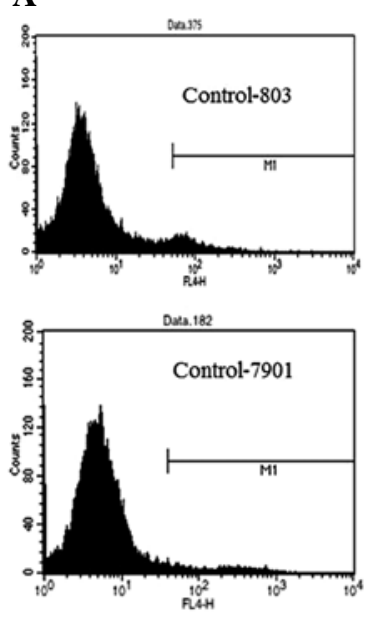
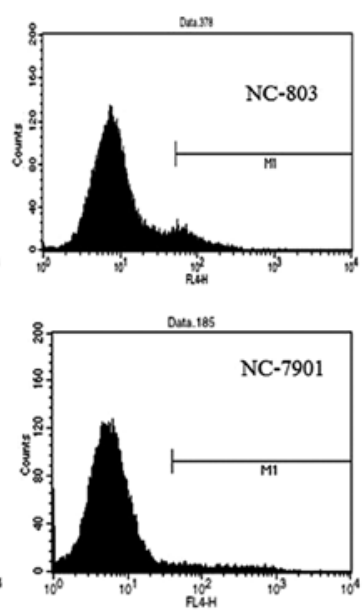
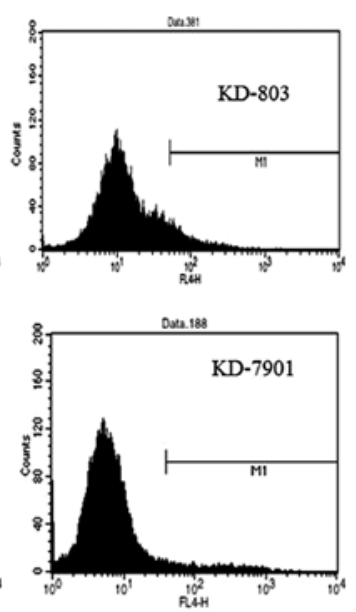

B

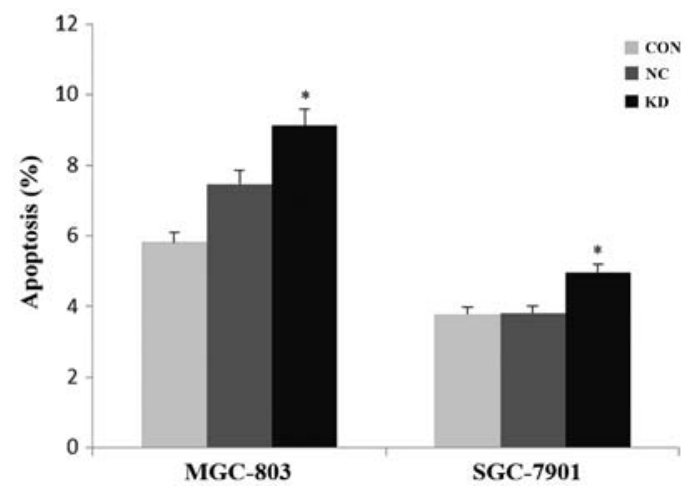

Figure 4. Knockdown of S100P promotes the apoptosis of GC cells. Negative control or lenti-S100P-shRNA-transduced MGC-803 and SGC-7901 cells were stained with Annexin V-APC and analyzed with FACS. The figure shows the results from three independent experiments. (A) Lenti-S100P-shRNA promotes apoptosis in MGC-803 and SGC-7901 cells. (B) Statistical analysis showed that knockdown of S100P expression promotes GC cell apoptosis compared to the control group (CON, uninfected cells; NC, negative control; KD, knockdown).

verified that knockdown of S100P by the lentiviral-mediated RNAi specifically promoted apoptosis of the GC cell lines, and S100P may be involved in the cell survival pathway.

Differential gene expression analysis by PCR array. To obtain further insight into the mechanism of S100P in gastric carcinoma cell apoptosis, the mRNA expression profile of S100P shRNA-transfected SGC-7901 cells was compared with that of the negative control using a human apoptosis PCR array containing 86 cell apoptosis-related genes. Table II shows the fold difference between the knockdown and negative control. Of the 86 apoptosis-associated genes in this array, 12 genes demonstrated at least a 1.5 -fold difference in expression between the two groups. Nine genes exhibited downregulation, while 3 genes were upregulated in the knockdown group. FOS, DDIT3 and FN1 were significantly elevated, while FASLG, DAPK1, CTNNB1 and CASP2 were notably downregulated before and after S100P silencing.

\section{Discussion}

Gastric cancer is one of the most common types of cancer and is the leading cause of death worldwide, with an incidence which varies greatly across different geographic locations. The American Cancer Society estimates that 21,320 cases of GC were diagnosed and 10,540 individuals died from GC in 2012 (15). More new cases of GC are diagnosed in China each year than in any other country. Previous research indicates that S1100P is involved in the development of various types of cancers. In the present study, we hypothesized that S100P serves as an oncogenic factor in GC development. Firstly, we measured S100P expression in GC tissues. The results showed that S100P was frequently expressed in GC tissues, with 54.2\% of cases demonstrating strong immunoreactivity, consistent with the findings of Parkkila et al (16). Ge et al (17) demonstrated that S100P was upregulated in GC in comparison with a normal control and was correlated with TNM stage and prognosis. Moreover, the 5-year survival rate was significantly lower in patients with high levels of S100P expression than in patients with low levels of expression. In contrast, Jia et al (18) reported decreased expression of S100P in GC compared to normal tissues. Patients with S100P-positive cancers showed a statistical better survival than patients with negative S100P expression. Liu et al (19) found that the expression of S100P between normal and cancerous tissues did not significantly differ. Nevertheless, the expression of S100P in GC is still in dispute at the present time. Therefore, investigation of more clinical samples, particularly GC, and their non-cancerous counterparts is required to conclude whether there is a difference in S100P expression and whether there is a correlation between S100P expression and clinicopathological features.

Lentiviral vectors with large coding capacity of transgene cassettes into dividing and quiescent cells have become some of the most widely used vectors for fundamental biological research, function genomics and gene therapy (20). Secondly, we employed a lentiviral short hairpin delivery system in our trial to infect and efficiently silence S100P in the GC cell lines. As shown in Fig. 2, we constructed the S100P shRNA lentivirus, which sufficiently knocked down the expression levels of S100P mRNA and protein in the GC cells compared with the negative control, thus, ensuring the credibility of the subsequent assays. We also confirmed that knockdown of S100P markedly inhibited the colony formation capacity of GC cells (Fig. 3). These results indicate that $\mathrm{S} 100 \mathrm{P}$ plays an important role in GC cell growth in the short or relative long term. We then performed apoptotic analysis by FACS, attempting to uncover the mechanism by which shRNA of S100P controls gastric cell growth. Our data revealed that S100P shRNA had a marked function on GC survival via induction of apoptosis (Fig. 4).

S100P has been found to protect NIH3T3 cells and pancreatic cancer cells from apoptosis induced by the cytotoxic agent 5-FU and from detachment from a solid substrate $(6,21)$. Overexpression of S100P in PC3 cells was found to promote cell growth, proliferation and reduced basal apoptosis rate. Furthermore, prostate cancer cells overexpressing S100P are protected against camptothecin-induced apoptosis (22). In 
hepatocellular carcinoma, S100P expression downregulated by RNA interference-mediated gene silencing increased the basal levels of apoptotic cells, which suggests that S100P may act as an aggressiveness factor in HCC cells by conferring resistance to basal apoptosis (23). These findings together with our results suggest that S100P serves as an important factor that contributes to the aggressive nature of cancer via inhibition of apoptosis. To date, however, the specific signaling pathway of S100P involved in GC cell apoptosis remains unclear.

To clarify the molecular mechanism by which S100P affects cell apoptosis, we employed the PCR array to profile the expression of apoptosis-associated genes. The result showed that 12 genes had a $>1.5$-fold difference in gene expression between the S100P shRNA-transfected cells and the negative control. Expression of 3 genes was significantly elevated, while 9 genes appear to be downregulated in the S100P-knockdown group.

Celecoxib inhibits cancer cell growth and stimulates apoptosis. Upregulation of S100P expression by celecoxib in GC cells was found to negatively affect the drug antitumorigenic activity through inhibition of apoptosis. Moreover, apoptosis induced by celecoxib was stimulated by a small interfering RNA targeting S100P. Yet, there was no significant difference in apoptotic cells between the siRNA S100P and non-silenced group (24). DDIT3 (also known as CHOP, GADD153), upregulated in the S100P-knockdown cells, is considered a key event in endoplasmic reticulum stress-mediated apoptosis (25). Expression of the ER stress-responsive transcription factor CHOP was found to be correlated with patient survival and remained an independent prognostic variable in pairwise comparisons with all clinical variables tested (26). CHOP expression had no statistical difference between the mock and S100P-overexpressing cells. Celecoxib-induced expression of CHOP mRNA was suppressed in S100P-overexpressing cells, which suggests that S100P induction inhibits celecoxibinduced apoptosis through suppression of CHOP expression (24). This finding is similar to our presumption that S100P affects apoptosis through dysregulation of the expression of CHOP. Consequently, our future research aims to confirm the protein level changes in genes with differential expression following S100P depletion and to collect sufficient GC samples with controls to detect S100P and validate gene expression and find a correlation between S100P and the validated genes in GC.

In conclusion, to the best of our knowledge, the present study proved for the first time that lentiviral-mediated RNAi targeting S100P promotes cell apoptosis and inhibits colony-formation ability of GC cells. Our data indicate that S100P serves as an oncogene in GC development. Therefore, knockdown of S100P expression by a lentiviral-delivered shRNA may be a potential and valuable strategy for the treatment of GC.

\section{References}

1. Garcia M, Jemal A, Ward EM, et al: Global Cancer facts \& Figures 2007. American Cancer Society, Atlanta, 2007.

2. Jemal A, Bray F, Center MM, Ferlay J, Ward E and Forman D: Global cancer statistics. CA Cancer J Clin 61: 69-90, 2011.

3. Sun XD, Mu R, Zhou YS, et al: Analysis of mortality rate of stomach cancer and its trend in twenty years in China. Zhonghua Zhong Liu Za Zhi 26: 4-9, 2004 (In Chinese).
4. Donato R: S100: a multigenic family of calcium-modulated proteins of the EF-hand type with intracellular and extracellular functional roles. Int J Biochem Cell Biol 33: 637-668, 2001.

5. Becker T, Gerke V, Kube E and Weber K: S100P, a novel $\mathrm{Ca}^{2+}$-binding protein from human placenta. cDNA cloning, recombinant protein expression and $\mathrm{Ca}^{2+}$-binding properties. Eur J Biochem 207: 541-547, 1992.

6. Arumugam T, Simeone DM, Golen KV and Logsdon CD: S100P promotes pancreatic cancer growth, survival, and invasion. Clin Cancer Res 11: 5356-5364, 2005.

7. Fuentes MK, Nigavekar SS, Arumugam T, Logsdon CD, Schmidt AM, Park JC and Huang EH: RAGE activation by S100P in colon cancer stimulates growth, migration, and cell signaling pathways. Dis Colon Rectum 50: 1230-1240, 2007.

8. Wang G, Platt-Higgins A, Carroll J, de Silva Rudland S, Winstanley J, Barraclough R and Rudland PS: Induction of metastasis by S100P in a rat mammary model and its association with poor survival of breast cancer patients. Cancer Res 66: 1199-1207, 2006.

9. Bartling B, Rehbein G, Schmitt WD, Hofmann HS, Silber RE and Simm A: S100A2-S100P expression profile and diagnosis of non-small cell lung carcinoma: impairment by advanced tumour stages and neoadjuvant chemotherapy. Eur J Cancer 43: 1935-1943, 2007.

10. Austermann J, Nazmi AR, Muller-Tidow C and Gerke V: Characterization of the $\mathrm{Ca}^{2+}$-regulated ezrin-S100P interaction and its role in tumor cell migration. J Biol Chem 283: 29331-29340, 2008.

11. Filipek A, Jastrzebska B, Nowotny M and Kuznicki J: CacyBP/ SIP, a calcyclin and Siah-1-interacting p rotein, binds EF-hand proteins of the S100 family. J Biol Chem 277: 28848-28852, 2002.

12. Shyu RY, Huang SL and Jiang SY: Retinoic acid increases expression of the calcium-binding protein S100P in human gastric cancer cells. J Biomed Sci 10: 313-319, 2003.

13. Allred DC, Harvey JM, Berardo M and Clark GM: Prognostic and predictive factors in breast cancer by immunohistochemical analysis. Mod Pathol 11: 155-168, 1998.

14. Livak KJ and Schmittgen TD: Analysis of relative gene expression data using real-time quantitative PCR and the 2(-Delta Delta C (T)) Method. Methods 25: 402-408, 2001.

15. Siegel R, Naishadham D and Jemal A: Cancer statistics, 2012. CA Cancer J Clin 62: 10-29, 2012.

16. Parkkila S, Pan PW, Ward A, et al: The calcium-binding protein $\mathrm{S} 100 \mathrm{P}$ in normal and malignant human tissues. BMC Clin Pathol 8: 2, 2008.

17. Ge F, Wang C, Wang W and Wu B: S100P predicts prognosis and drug resistance in gastric cancer. Int $\mathbf{J}$ Biol Markers 28: e387-e392, 2013.

18. Jia SQ, Niu ZJ, Zhang LH, et al: Identifcation of prognosisrelated proteins in advanced GC by mass spectrometry-based comparative proteomics. J Cancer Res Clin Oncol 135: 403-411, 2009.

19. Liu J, Li X, Dong GL et al: In silico analysis and verification of S100 gene expression in gastric cancer. BMC Cancer 8: 261, 2008.

20. Mátrai J, Chuah MK and VandenDriessche T: Recent advances in lentiviral vector development and applications. Mol Ther 18: 477-490, 2010.

21. ArumugamT, Simeone DM, Schmidt AM and Logsdon CD: S100P stimulates cell proliferation and survival via receptor for advanced glycation end products (RAGE). J Biol Chem 279: 5059-5065, 2004.

22. Basu GD, Azorsa DO, Kiefer JA, et al: Functional evidence implicating S100P in prostate cancer progression. Int J Cancer 123: 330-339, 2008.

23. Kim JK, Jung KH, Noh JH, et al: Targeted disruption of S100P suppresses tumor cell growth by downregulation of cyclin D1 and CDK2 in human hepatocellular carcinoma. Int J Oncol 35: 1257-1264, 2009.

24. Namba T, Homan T, Nishimura T, Mima S, Hoshino T and Mizushima T: Up-regulation of S100P expression by nonsteroidal anti-inflammatory drugs and its role in anti-tumorigenic effects. J Biol Chem 284: 4158-4167, 2009.

25. Oyadomari S and Mori M: Roles of CHOP/GADD153 in endoplasmic reticulum stress. Cell Death Differ 11: 381-389, 2004.

26. Dalton LE, Clarke HJ, Knight J, et al: The endoplasmic reticulum stress marker CHOP predicts survival in malignant mesothelioma. Br J Cancer 108: 1340-1347, 2013. 